Gut and Liver, Vol. 13 No. 4, July 2019, pp. 394-401

\title{
Clinical Application of Circulating Tumor Cells in Gastric Cancer
}

\author{
Moon Won Lee, Gwang Ha Kim, Hye Kyung Jeon, and Su Jin Park \\ Department of Internal Medicine, Pusan National University School of Medicine, and Biomedical Research Institute, Pusan National University \\ Hospital, Busan, Korea
}

Early detection and accurate monitoring of cancer is important for improving clinical outcomes. Endoscopic biopsy and/ or surgical resection specimens are the gold standard for diagnosing gastric cancer and are also useful for selecting therapeutic strategies based on the analysis of genomic/ immune parameters. However, these approaches cannot be easily performed because of their invasiveness and because these specimens do not always reflect tumor dynamics and drug sensitivities during therapeutic processes, especially chemotherapy. Accordingly, many researchers have tried to develop noninvasive novel biomarkers that can monitor realtime tumor dynamics for early diagnosis, prognostic evaluation, and prediction of recurrence and therapeutic efficacy. Circulating tumor cells (CTCs) are metastatic cells that are released from the primary tumors into the blood stream and comprise a crucial step in hematogenous metastasis. CTCs, as a liquid biopsy, have received a considerable amount of attention from researchers since they are easily accessible in peripheral blood, avoiding the invasiveness associated with traditional biopsy techniques; they can also be used to derive clinical information for monitoring disease status. In this review, with respect to CTCs, we summarize the metastatic cascade, detection methods, clinical applications, and prospects for patients with gastric cancer. (Gut Liver 2019;13:394401)

Key Words: Circulating tumor cells; Liquid biopsy; Neoplasms; Stomach neoplasms

\section{INTRODUCTION}

Gastric cancer (GC) is the 6th most prevalent cancer $(1,033,701$ new cases per year) and the 2nd most common cause of cancerrelated deaths worldwide in 2018. ${ }^{1}$ Despite the development of diagnostic and therapeutic modalities, more than $40 \%$ of patients with advanced GC have a poor prognosis and exhibit no response to chemotherapy or resistance to chemotherapy during treatment. ${ }^{2}$ Approximately 30\% to $40 \%$ of GC patients experience tumor recurrence and metastasis even after curative resection. ${ }^{3,4}$ This suggests the presence of potential metastatic cells that cannot be detected based on conventional diagnostic methods.

Traditional tumor markers such as carcinoembryonic antigen (CEA) and cancer antigen 19-9 (CA19-9) are known biomarkers of GC. However, the positivity rate of these markers is less than $40 \%$ in GC patients, and the sensitivity and specificity of assays to detect these markers are insufficient for diagnostic and prognostic approaches. ${ }^{5}$ Generally, the diagnosis of GC is based on endoscopic biopsy and/or surgical resection specimens, but these approaches cannot be easily performed due to their invasiveness. Furthermore, these specimens do not represent the intratumoral heterogeneity and mechanisms of tumor progression and resistance to treatment. Therefore, the development of new modalities that can predict recurrence and metastasis, and which can be used for diagnosis and evaluation of therapeutic response, is important to improve clinical outcomes for GC patients. $^{6}$

Circulating tumor cells (CTCs) are disseminated tumor cells in the peripheral blood of cancer patients. The first report of CTCs occurred in 1869 from Thomas Ashworth, who described the presence of tumor-derived epithelial cells in the blood compartment of a patient with end-stage metastatic cancer. ${ }^{7}$ However, because the number of cancer cells in circulation ranges from only approximately around $1-1,000$ cells $/ 10 \mathrm{~mL},{ }^{8}$ conventional methods are not adequate for their detection. Recently, numerous new approaches have been established to isolate, enumerate, and characterize CTCs in patients with various cancers. ${ }^{9}$ CTCs have strengths such as accessibility and superiority over

Correspondence to: Gwang Ha Kim

Department of Internal Medicine, Pusan National University School of Medicine, and Biomedical Research Institute, Pusan National University Hospital, 179 Gudeok-ro, Seo-gu, Busan 49241, Korea

Tel: +82-51-240-7869, Fax: +82-51-244-8180, E-mail: doc0224@pusan.ac.kr

Received on October 30, 2018. Revised on November 19, 2018. Accepted on November 23, 2018. Published online February 27, 2019 pISSN 1976-2283 eISSN 2005-1212 https://doi.org/10.5009/gnl18484

@ This is an Open Access article distributed under the terms of the Creative Commons Attribution Non-Commercial License (http://creativecommons.org/licenses/by-nc/4.0) which permits unrestricted non-commercial use, distribution, and reproduction in any medium, provided the original work is properly cited. 
conventional tumor markers; moreover, based on the analysis of CTCs, researchers can elucidate genetic and phenotypic differences between primary and metastatic tumors. Herein, we summarize the metastatic cascade associated with CTCs, provide an overview of the detection methods for CTCs, and suggest clinical applications for CTCs in GC patients; we then discuss their future use in the clinical practice.

\section{CIRCULATING TUMOR CELLS AND METASTASIS}

Cancer metastasis is a multi-step process that includes the loss of intercellular connections, invasion into the basal membrane and surrounding tissues, intravasation into venous or lymphatic vessels, which generates CTCs, survival in the peripheral system, extravasation, and proliferation at secondary sites. ${ }^{10-12}$ Epithelial tumor cells are immobile due to strong cell-to-cell and cell-toextracellular adhesions, which consist of adherent junctions, tight junctions, and desmosomes. Moreover, conditions in the blood stream are too harsh for the survival of epithelial tumor cells. Therefore, these cells undergo epithelial-to-mesenchymal transition (EMT), which is associated with the loss of adhesion, increased plasticity, and the capacity for migration and invasion. EMT is a molecular and cellular change that includes the down-regulation of epithelial proteins such as E-cadherin, claudins and cytokeratins and the up-regulation of mesenchymal proteins such as $\mathrm{N}$-cadherin, fibronectin and vimentin, which confers increased motility and invasiveness to the cells. ${ }^{10-12}$ EMT promotes the generation of CTCs by increasing tumor cell invasiveness, ${ }^{13-15}$ promoting tumor cell intravasation, ${ }^{16,17}$ and facilitating tumor cell survival in the peripheral system. ${ }^{18,19}$

The correlation between EMT and tumor cell invasiveness or CTCs has been confirmed, but the details of the metastatic cascade are still controversial. Two hypotheses have been proposed for the metastatic model associated with EMT: EMT/mesenchymal-epithelial migration (MET) model and collective migration model (Fig. 1). ${ }^{20}$ In the EMT/MET model, at first, epithelial cancer cells must undergo EMT to become invasive and motile and to generate CTCs; after that, CTCs circulate throughout the entire body and extravasate to distant secondary sites. After extravasation to secondary sites, cancer cells need to recover their epithelial properties via MET, the inverse process of EMT, and then finally colonize at the distant site to complete the metastasis. ${ }^{21-24}$ This metastatic model is the first reported and most widely known model, and also explains why the histological characteristics of the primary tumor are consistent with those of the secondary tumor. ${ }^{22}$ Furthermore, recent studies have reported a switch between EMT and MET marker phenotypic plasticity in some cancer types. ${ }^{25,26}$ In the collective migration model, epithelial-like and mesenchymal-like cancer cells can cooperate during collective migration to achieve metastasis instead of undergoing the difficult process of EMT and subsequent MET. ${ }^{27,28}$ Instead of migrating as a single cell, cancer cells undergoing various degrees of EMT coexist as multi-cellular clusters that migrate collectively. More motile, invasive mesenchymal-like cells aggregate at the invasive front of multi-cellular clusters to facilitate this process, whereas epithelial-like cells follow behind and exploit the opportunity to proliferate and colonize at the distant sites after extravasation. ${ }^{28}$ Accordingly, multi-cellular cancer fragments, or so-called tumor micro-emboli or CTC clusters, have been observed and correlate with distant metastasis. $^{29,30}$ Of note, the EMT/MET and collective migration models might not be independent or opposing. Notably, tumor cells might use both mechanisms alternately under certain circumstances, or the two mechanisms could synergistically contribute to metastases.

\section{METHODS TO DETECT CICRULATING TUMOR CELLS}

In general, CTCs are rare in the peripheral blood of cancer patients. The enumeration of these CTCs is a great technological challenge. Thus, the enrichment methods require sufficient sensitivity and reproducibility. Current methods for separating CTCs include those based on immunoaffinity differences, using an antibody that binds to a specific protein marker on the surface of cancer cells, ${ }^{31-33}$ and differences in physical properties such as the sizes, densities, and electrical characteristics of cancer cells and blood cells (Fig. 2). ${ }^{34-39}$

Immunoaffinity-based separation is the most widely-used strategy. This method uses specific biomarkers such as epithelial cell adhesion molecule (EpCAM) and CD45 which are expressed on the cell surface, to capture cells. The antibodies used for selection are typically linked to the surface of a device or to a magnetic substance allowing them to be captured by a magnetic field (i.e., immunomagnetic capture). During positive selection, tumor-associated cell surface antigens such as EpCAM are targeted, whereas negative selection removes background cells by

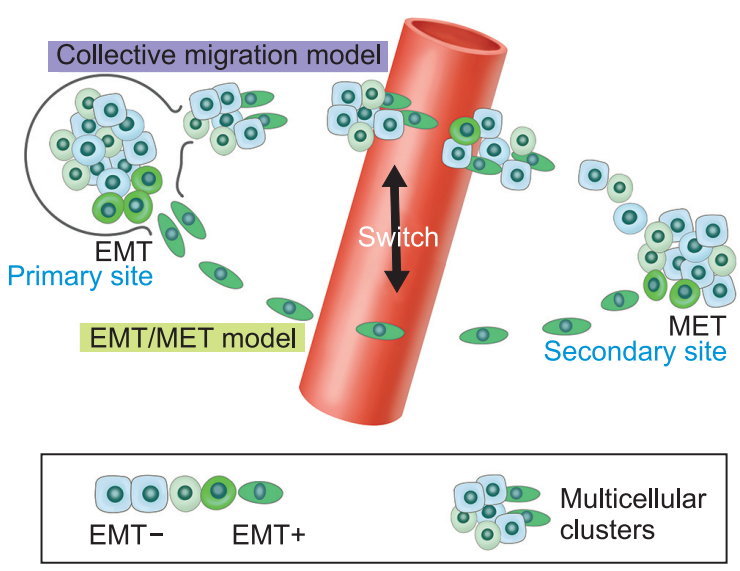

Fig. 1. Epithelial-mesenchymal transition (EMT) and mesenchymalepithelial transition (MET) in the emergence and progression of carcinoma. Adapted from Jie XX, et al. Oncotarget 2017;8:81558-81571. ${ }^{20}$ 


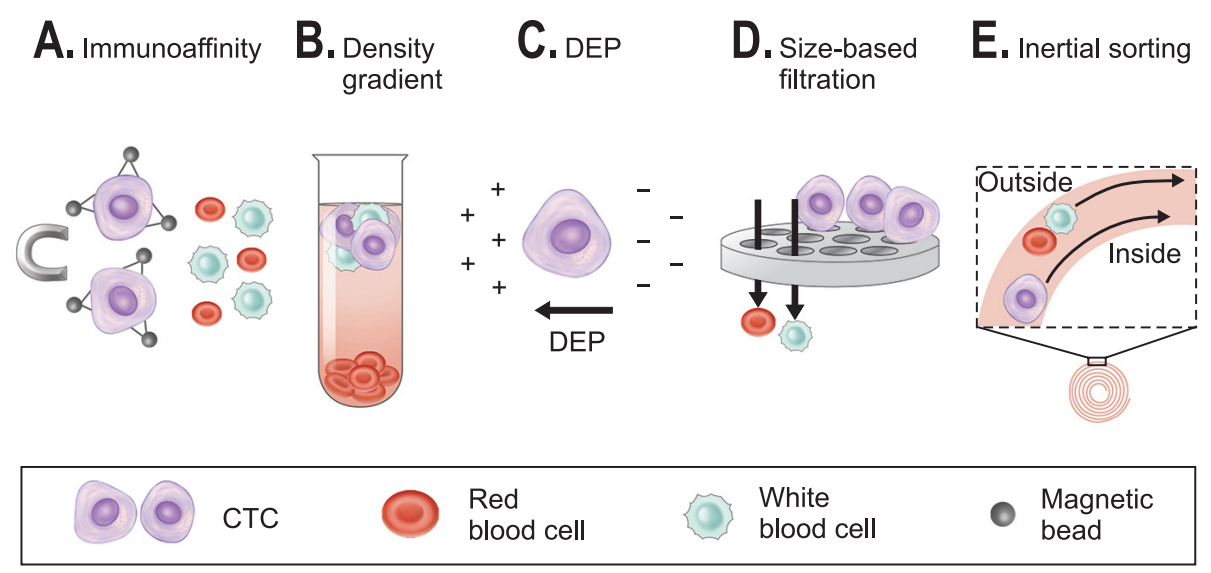

Fig. 2. Enrichment technologies for circulating tumor cells (CTCs). A: Immunoaffinity method using immunomagnetic particles that bind to the CTC surface antigen. B: Density gradient method separating other blood cells from CTCs based on their density. C: Dielectrophoresis (DEP) utilizing the dielectric properties of CTCs under an electric field. D: Size-based filtration resulting in enrichment based on the larger size of CTCs compared with the sizes of other cells. E: Inertia sorting method using Dean force at microfluidic channels. Adapted from Alix-Panabières C and Pantel K. Nat Rev Cancer 2014;14:623-631..$^{39}$

antigens not expressed on CTCs (e.g., CD45). Specifically, the CellSearch $^{\mathrm{TM}}$ platform (Veridex LLC, Huntingdon Valley, PA, USA) was approved by the U.S. Food and Drug Administration (FDA) for the diagnosis and prognostic evaluation of patients with metastatic breast cancer, colorectal cancer, and prostate cancer between 2004 and 2008. ${ }^{40}$ However, the expression level of CTCs is extremely variable, and CTCs with low target antibody expression are more difficult to capture. ${ }^{41}$ Moreover, separation processes for CTCs include complex steps and are time consuming with respect to achieving sufficient reactions between antigens and antibodies. In addition, the heterogeneous array of surface markers expressed by CTCs has made it impossible to identify a universal CTC-specific antigen.

In the density gradient method, blood samples are diluted and laid over the media, and then separated by centrifugation. ${ }^{42}$ With this, it is easy to remove red blood cells with a high density, but it is difficult to separate CTCs and white blood cells due to their similar densities. Size-based filtration has been used to enrich CTCs based on the fact that they are larger than other blood cells. However, CTCs are generally 8 to $20 \mu \mathrm{m}$ in size, whereas leukocytes are 6 to $10 \mu \mathrm{m}$; thus, leukocytes will be present to some extent in samples treated by the size-based separation methods, which makes it difficult to capture high-purity CTCs. ${ }^{43}$ In the inertia sorting method, the CTCs are isolated using a spiral channel microfluidic chip, which separates differentsized cells with different Dean forces in a curved channel. With this, it is possible to capture CTCs exhibiting different expression levels of specific surface proteins, and this technique has several advantages such as fast separation time, ease of use, and low cost. However, size-based filtration has the disadvantage of potentially capturing contaminated cells. ${ }^{44}$

Dielectrophoresis (DEP) is an electrokinetic method that ex- ploits the dielectric properties of floating cells for identification and separation. This method isolates CTCs based on different responses to DEP due to differences in size and membrane properties. Further, it is advantageous for the separation of CTCs because of its simple experimental set-up, much higher operational flow rate compared to other separator methods, and lack of dependence on cell surface markers. ${ }^{45}$ However, for efficient isolation, whole blood cannot be used directly; there is also limitations associated with the use of a special buffer optimized for electrical conductivity to increase the efficiency of cancer cell isolation.

With advances in technology, next generation microfluidic platforms that enrich CTCs have been developed for cost-effectiveness and high levels of purity. Therefore, if these platforms with high sensitivity and specificity can be further developed, they could be used extensively in clinical practice as well as in the studies on CTCs.

\section{CLINICAL DATA REGARDING CIRCULATING TUMOR CELLS IN PATIENTS WITH GASTRIC CANCER}

\section{Diagnostic and prognostic value of circulating tumor cells}

Based on a meta-analysis of the diagnostic accuracy of various CTC detection methods, the pooled sensitivity and specificity of CTCs for GC were 42\% (95\% confidence interval [CI], 21\% to $67 \%$ ) and $99 \%$ (95\% CI, 96\% to $100 \%$ ), respectively; ${ }^{46}$ the authors of this study concluded that CTC detection has a limited role in screening test for GC, but might be used as a non-invasive method for the confirmation of GC diagnosis. Seventeen of 20 studies included in this meta-analysis used reverse transcriptase-polymerase chain reaction (RT-PCR) for cytokeratin or CEA 
to detect CTCs and the results varied among different detection methods. RT-PCR is one molecular approach for the detection of CTCs; however, an ideal method should focus on the tumor cells directly rather than indirectly-linked surrogate markers via a molecular approach. ${ }^{47}$ To date, the CellSearch ${ }^{\mathrm{TM}}$ system (Veridex LLC) is the only CTC detection technique approved by the U.S. FDA. Using this platform, it is possible to obtain highly reproducible quantitative results in different laboratories. Therefore, this review will focus further on studies reporting significant data regarding the role of CTCs in GC that were obtained by the cytometric methods including the CellSearch ${ }^{\mathrm{TM}}$ system. ${ }^{48-60}$ The results of these studies are summarized in Table $1 .^{61}$

In our previous study, ${ }^{56}$ CTCs were measured in 116 GC patients and 31 healthy individuals using a centrifugal microfluidic system with a new fluid-assisted separation technique, specifically based on size-selective CTC isolation. ${ }^{56}$ When using a CTC level of $\geq 2$ per $7.5 \mathrm{~mL}$ of blood, the sensitivity and specificity for differentiating GC patients from healthy controls was 85.3\% (95\% CI, $81.4 \%$ to $87.2 \%$ ) and 90.3\% (95\% CI, 75.6\% to 97.4\%), respectively. In addition, CTCs were detected in more than $80 \%$ of early stage (T1 or N0) GC patients. Although CTCs were not associated with any clinicopathologic features such as staging, histologic type, or mucin phenotype, our results suggest that CTCs could be an early diagnostic biomarker for GC. In a recent study, using a novel wedge-shaped microfluidic chip, CTCs were detected in 75\% (30/40) of GC patients, whereas CTCs were not detected in 25 healthy donors. ${ }^{60}$ In this study, CTCs were associated with tumor differentiation, lymphovascular invasion, and staging. These differences in the association between CTCs and clinicopathologic characteristics might be attributed to heterogeneity in the baseline clinicopathologic characteristics and the use of different CTC detection methods.

Table 1. Summary of Studies on Circulating Tumor Cells Using Cytometric Methods in Patients with Gastric Cancer

\begin{tabular}{|c|c|c|c|c|c|c|c|c|}
\hline Year & Author & $\begin{array}{c}\text { No. of } \\
\text { patients }\end{array}$ & Detection methods & Markers & $\begin{array}{l}\text { Positive } \\
\text { definition }\end{array}$ & $\begin{array}{l}\text { CTC detection, } \\
\text { No. }(\%)\end{array}$ & Outcomes & $\begin{array}{c}\text { Clinical } \\
\text { significance }\end{array}$ \\
\hline 2007 & $\begin{array}{l}\text { Pituch-Noworolska } \\
\text { et al. }\end{array}$ & 57 & FACS & CK8,18,19 & $\geq 3$ CTCs & $31(54)$ & OS & No \\
\hline \multirow[t]{2}{*}{2008} & Hiraiwa et al. ${ }^{49}$ & Non-metastatic, 14 & CellSearch & EpCAM & $\geq 2$ CTCs & $2(14)$ & OS & Yes \\
\hline & & Metastatic, 27 & & CK8,18,19 & & $15(56)$ & Monitoring & \\
\hline \multirow[t]{3}{*}{2010} & Matsusaka et al..$^{50}$ & Baseline, 52 & CellSearch & EpCAM & $\geq 4$ CTCs & $17(33)$ & OS, PFS & Yes \\
\hline & & 2-wk, 51 & & CK8,18,19 & & $7(14)$ & Monitoring & \\
\hline & & 4-wk, 48 & & & & $9(19)$ & & \\
\hline \multirow[t]{2}{*}{2013} & Uenosono et al. ${ }^{51}$ & Resection, 148 & CellSearch & EpCAM & $\geq 1 \mathrm{CTC}$ & $16(11)$ & OS, RFS & Yes \\
\hline & & Non-resectable, 103 & & CK8,18,19 & & $62(60)$ & & \\
\hline \multirow[t]{2}{*}{2015} & Okabe et al. ${ }^{52}$ & 136 & CellSearch & EpCAM & $\geq 1 \mathrm{CTC}$ & $25(18)$ & PFS & Yes \\
\hline & & & & CK8,18,19 & & & & \\
\hline 2015 & Yuan et al. ${ }^{53}$ & 31 & FACS & CD44+ CD45- & - & $14(45)$ & - & - \\
\hline \multirow[t]{3}{*}{2016} & Kolostova et al. ${ }^{54}$ & 22 & MetaCell & CK7,18,19, 20 & - & $13(59)$ & - & - \\
\hline & & & & EpCAM, MUC1, & & & & \\
\hline & & & & HER2, EGFR & & & & \\
\hline \multirow[t]{2}{*}{2016} & Li et al. ${ }^{55}$ & Baseline, 136 & CellSearch & EpCAM & $\geq 3$ CTCs & $57(42)$ & OS, PFS & Yes \\
\hline & & 6-wk, 106 & & CK8,18,19 & & $26(25)$ & Monitoring & \\
\hline 2017 & Kang et $a l^{56}$ & 116 & FAST & EpCAM/CK & $\geq 2$ CTCs & $99(85)$ & - & - \\
\hline \multirow[t]{2}{*}{2017} & Liu et $a .^{57}$ & 59 & CELLection & EpCAM & $>2$ CTCs & $36(61)$ & OS, PFS & Yes \\
\hline & & & & CK7,8,18,19 & & & Monitoring & \\
\hline \multirow[t]{2}{*}{2017} & Pernot et al. ${ }^{58}$ & Baseline, 106 & CellSearch & EpCAM & $\geq 2$ CTCs & $49(46)$ & OS, PFS & Yes \\
\hline & & 4-wk, 65 & & CK8,18,19 & & $12(18)$ & Monitoring & \\
\hline \multirow[t]{2}{*}{2018} & Brungs et al. ${ }^{59}$ & 43 & IsoFlux & EpCAM & $\geq 17$ CTCs & $20(47)$ & OS & Yes \\
\hline & & & platform & & & & Monitoring & \\
\hline 2018 & Yang et al..$^{60}$ & 40 & Microfluidic chip & CK & $\geq 1 \mathrm{CTC}$ & $20(75)$ & - & Yes \\
\hline
\end{tabular}

CTC, circulating tumor cell; FACS, fluorescence-activated cell sorting; CK, cytokeratin; OS, overall survival; EpCAM, epithelial cell adhesion molecule; PFS, progression-free survival; RFS, recurrence-free survival; HER2, human epidermal growth factor receptor 2; EGFR, epidermal growth factor receptor; FAST, fluid-assisted separation technique. Adapted from Jeon HK and Kim GH. Korean J Helicobacter Up Gastrointest Res 2018;18:162-167. ${ }^{61}$ 
The measurement of CTCs in GC patients can be useful for predicting patient survival and prognosis. In a prospective study including 251 patients with advanced GC using the CellSearch ${ }^{\mathrm{TM}}$ system, CTCs were detected in 16 patients $(16 / 148,10.8 \%)$ in the resection group with a higher relapse rate and in 62 patients $(62 / 103,60.2 \%)$ in the non-resectable group, but were not detected in healthy volunteer. Thus, the evaluation of CTCs might be a useful strategy to predict tumor progression and prognosis in GC patients. Based on a meta-analysis (including 26 studies comprising 2,566 GC patients) evaluating the prognostic significance of CTCs in GC patients, the frequency of CTC detection was higher in advanced GC than early GC, in poorly differentiated GC than well/moderately differentiated GC, and in GC with lymphatic metastasis than that without lymphatic metastasis; it was also significantly related to the disease-free and overall survival of patients. ${ }^{62}$

In addition to being diagnostic and prognostic indicators, CTCs can be used to monitor chemotherapy response. The measurement of CTCs using peripheral blood is non-invasive and can be performed repeatedly. Thus, periodically monitoring CTCs could be helpful to predict the efficacy of chemotherapeutics. Although CEA and CA19-9 are frequently used as markers in GC, they are not appropriate for this application because temporary increases can be detected after chemotherapy and many patients with advanced GC have normal levels of CEA and CA19-9. Matsusaka et al..$^{50}$ measured CTC levels at baseline, 2 weeks, and 4 weeks in 52 patients with advanced GC undergoing S-1-based chemotherapy. Patients with $\geq 4$ CTCs at 2- and 4-weeks post-chemotherapy had a shorter median progressionfree survival (PFS) and overall survival (OS). ${ }^{50}$ In another study measuring CTC levels at baseline and after 6 weeks of chemotherapy in 136 patients with advanced GC, patients with $\geq 3$ CTCs after chemotherapy had a shorter PFS and OS; moreover, an elevated number of CTCs after chemotherapy corresponded to ineffective therapeutic responses. ${ }^{55}$ Therefore, monitoring dynamic changes in CTCs in response to therapy might be a useful alternative for assessing treatment resistance in GC patients.

\section{Value of circulating tumor cells for selecting therapeutic agents}

Overcoming tumor heterogeneity is a major challenge for the personalized treatment of GC. Therefore, the further characterization of CTCs might help to identify specific morphological, phenotypic, and molecular features of different cancer types based on time, disease stage, and therapeutic definitions. ${ }^{63}$ As stated earlier, CTCs that have undergone the EMT exhibit enhanced cell motility rather than growth, resulting in the ability of cancer cells to exit the vasculature. In this situation, most CTCs are not responsive to cytotoxic chemotherapy because they do not undergo cell division. ${ }^{64,65}$ Therefore, the addition of CTC-targeted treatment to conventional chemotherapy might be helpful to improve prognosis for cancer patients. In non-small- cell lung cancers, a therapeutic agent targeting an epidermal growth factor receptor (EGFR) mutation has been developed, therefore, it is important to also detect this EGFR mutation in CTCs. Currently, there are several clinical reports suggesting that detection rate of the EGFR mutation in the CTCs of patients with non-small-cell lung cancer is comparable to that in tissues, confirming a high rate of concordance. ${ }^{66-68}$

For GC, trastuzumab, which bind the human epidermal growth factor receptor 2 (HER2) gene, and ramucirumab, which bind vascular endothelial growth factor receptor-2, have been developed. Especially, the overexpression of HER2 is a selective biomarker for treatment with the monoclonal antibody bevacizumab in metastatic GC. Recently, Iwatsuki et al. ${ }^{69}$ evaluated CTCs and their HER2 status in patients with gastrointestinal cancer. Among the 62 CTC-positive cases, 22 (35.5\%) showed discordance in terms of HER2 status between primary tumors and CTCs. Among the HER2-negative primary tumors, 17 of 54 developed HER2-positive CTCs, and among the HER2-positive primary tumors, five of eight were associated with HER2negative CTCs. These results suggest that for primary HER2negative tumors, CTCs could acquire HER2 gene amplification during cancer progression, and therefore, it might be necessary to determine the HER2 status of CTCs, as a liquid biopsy, to suggest personalized treatment strategies for GC. Thus, it is expected that CTCs derived from GC patients could be used to predict responses to targeted therapy. These CTCs require precise medicine, which will not only help to select the appropriate chemotherapy but will also allow for the periodic monitoring of mutations associated with resistance.

\section{FUTURE DIRECTIONS}

For the use of CTCs to become a clinical analytical method that complements tissue biopsies, there are many issues that need to be solved. Regarding the CellSearch ${ }^{\mathrm{TM}}$ system, cells that are positive for EpCAM are isolated, which can be considered a false negative for CTCs undergoing EMT. Therefore, it is necessary to find markers to accurately detect CTCs with an EMT phenotype. In addition, it is difficult to directly compare the sensitivity, specificity, and reproducibility of CTC separation methods because the types and preparations of various CTC isolation methods currently under development are different and have not been standardized. Therefore, it is important to develop a standardized isolation method to establish appropriate criteria for the use of CTCs as diagnostic and prognostic indicators of GC, and to confirm their clinical significance through large scale prospective clinical studies.

\section{CONCLUSIONS}

Technology associated with liquid biopsy was globally recognized as one of the world top 10 technologies in 2017 and com- 
prises a highly competitive field for the development of methods that enable the most simple and accurate diagnosis of cancer. Recently, several studies have shown that peripheral blood CTCs are useful to predict prognosis and monitor therapy in GC patients. Future CTC studies are expected to provide additional information to identify mechanisms that are important for the proliferation and metastasis of malignant tumors. Furthermore, these studies are expected to play a major role in precision medicine by uncovering molecular biologic targets related to drug sensitivity and resistance in GC patients.

\section{CONFLICTS OF INTEREST}

No potential conflict of interest relevant to this article was reported.

\section{ACKNOWLEDGEMENTS}

This work was supported by a Busan Cancer Center Research Grant (2018), Pusan National University Hospital.

\section{ORCID}

Moon Won Lee

Gwang Ha Kim

Hye Kyung Jeon

Su Jin Park https://orcid.org/0000-0002-8411-6398 https://orcid.org/0000-0001-9721-5734 https://orcid.org/0000-0002-1550-7192 https://orcid.org/0000-0001-9880-9825

\section{REFERENCES}

1. Bray F, Ferlay J, Soerjomataram I, Siegel RL, Torre LA, Jemal A. Global cancer statistics 2018: GLOBOCAN estimates of incidence and mortality worldwide for 36 cancers in 185 countries. CA Cancer J Clin 2018;68:394-424.

2. Hartgrink HH, Jansen EP, van Grieken NC, van de Velde CJ. Gastric cancer. Lancet 2009;374:477-490.

3. Lim DH, Kim DY, Kang MK, et al. Patterns of failure in gastric carcinoma after D2 gastrectomy and chemoradiotherapy: a radiation oncologist's view. Br J Cancer 2004;91:11-17.

4. Marrelli D, De Stefano A, de Manzoni G, Morgagni P, Di Leo A, Roviello F. Prediction of recurrence after radical surgery for gastric cancer: a scoring system obtained from a prospective multicenter study. Ann Surg 2005;241:247-255.

5. Li Y, Yang Y, Lu M, Shen L. Predictive value of serum CEA, CA199 and CA72.4 in early diagnosis of recurrence after radical resection of gastric cancer. Hepatogastroenterology 2011;58:21662170.

6. Kang EA, Han YM, Park JM, Yoo IK, Hong SP, Hahm KB. Liquid biopsy: current status and future perspective in gastric cancer and helicobacter infection. Korean J Helicobacter Up Gastrointest Res 2018;18:150-156.

7. Ashworth T. A case of cancer in which cells similar to those in the tumours were seen in the blood after death. Aust Med J 1869;14:146.

8. Nieva JJ, Kuhn P. Fluid biopsy for solid tumors: a patient's companion for lifelong characterization of their disease. Future Oncol 2012;8:989-998.

9. Pantel K, Brakenhoff RH. Dissecting the metastatic cascade. Nat Rev Cancer 2004;4:448-456.

10. Kalluri R, Weinberg RA. The basics of epithelial-mesenchymal transition. J Clin Invest 2009;119:1420-1428.

11. Thiery JP. Epithelial-mesenchymal transitions in tumour progression. Nat Rev Cancer 2002;2:442-454.

12. Thiery JP, Acloque H, Huang RY, Nieto MA. Epithelial-mesenchymal transitions in development and disease. Cell 2009;139:871890.

13. Liberko M, Kolostova K, Bobek V. Essentials of circulating tumor cells for clinical research and practice. Crit Rev Oncol Hematol 2013;88:338-356.

14. Derksen PW, Liu X, Saridin F, et al. Somatic inactivation of Ecadherin and p53 in mice leads to metastatic lobular mammary carcinoma through induction of anoikis resistance and angiogenesis. Cancer Cell 2006;10:437-449.

15. Hulit J, Suyama K, Chung S, et al. N-cadherin signaling potentiates mammary tumor metastasis via enhanced extracellular signal-regulated kinase activation. Cancer Res 2007;67:3106-3116.

16. Qi J, Wang J, Romanyuk O, Siu CH. Involvement of Src family kinases in N-cadherin phosphorylation and beta-catenin dissociation during transendothelial migration of melanoma cells. Mol Biol Cell 2006;17:1261-1272.

17. Drake JM, Strohbehn G, Bair TB, Moreland JG, Henry MD. ZEB1 enhances transendothelial migration and represses the epithelial phenotype of prostate cancer cells. Mol Biol Cell 2009;20:22072217.

18. Singh A, Settleman J. EMT, cancer stem cells and drug resistance: an emerging axis of evil in the war on cancer. Oncogene 2010;29:4741-4751.

19. Tiwari N, Gheldof A, Tatari M, Christofori G. EMT as the ultimate survival mechanism of cancer cells. Semin Cancer Biol 2012;22:194-207.

20. Jie XX, Zhang XY, Xu CJ. Epithelial-to-mesenchymal transition, circulating tumor cells and cancer metastasis: mechanisms and clinical applications. Oncotarget 2017;8:81558-81571.

21. Hugo H, Ackland ML, Blick T, et al. Epithelial: mesenchymal and mesenchymal. Epithelial transitions in carcinoma progression. J Cell Physiol 2007;213:374-383.

22. Chaffer CL, Thompson EW, Williams ED. Mesenchymal to epithelial transition in development and disease. Cells Tissues Organs 2007;185:7-19.

23. Chaffer CL, Weinberg RA. A perspective on cancer cell metastasis. Science 2011;331:1559-1564.

24. Banyard J, Bielenberg DR. The role of EMT and MET in cancer dissemination. Connect Tissue Res 2015;56:403-413.

25. Brabletz T, Jung A, Reu S, et al. Variable beta-catenin expression 
in colorectal cancers indicates tumor progression driven by the tumor environment. Proc Natl Acad Sci U S A 2001;98:1035610361.

26. Hudson LG, Zeineldin R, Stack MS. Phenotypic plasticity of neoplastic ovarian epithelium: unique cadherin profiles in tumor progression. Clin Exp Metastasis 2008;25:643-655.

27. Friedl P, Wolf K. Tumour-cell invasion and migration: diversity and escape mechanisms. Nat Rev Cancer 2003;3:362-374.

28. Friedl P, Gilmour D. Collective cell migration in morphogenesis, regeneration and cancer. Nat Rev Mol Cell Biol 2009;10:445-457.

29. Chui MH. Insights into cancer metastasis from a clinicopathologic perspective: epithelial-mesenchymal transition is not a necessary step. Int J Cancer 2013;132:1487-1495.

30. Wang C, Mu Z, Chervoneva I, et al. Longitudinally collected CTCs and CTC-clusters and clinical outcomes of metastatic breast cancer. Breast Cancer Res Treat 2017;161:83-94.

31. Riethdorf S, Fritsche H, Müller V, et al. Detection of circulating tumor cells in peripheral blood of patients with metastatic breast cancer: a validation study of the CellSearch system. Clin Cancer Res 2007;13:920-928.

32. Miller MC, Doyle GV, Terstappen LW. Significance of circulating tumor cells detected by the CellSearch system in patients with metastatic breast colorectal and prostate cancer. J Oncol 2010;2010:617421.

33. Harb W, Fan A, Tran T, et al. Mutational analysis of circulating tumor cells using a novel microfluidic collection device and qPCR assay. Transl Oncol 2013;6:528-538.

34. Desitter I, Guerrouahen BS, Benali-Furet N, et al. A new device for rapid isolation by size and characterization of rare circulating tumor cells. Anticancer Res 2011;31:427-441.

35. Kim TH, Lim M, Park J, et al. FAST: size-selective, clog-free isolation of rare cancer cells from whole blood at a liquid-liquid interface. Anal Chem 2017;89:1155-1162.

36. Gogoi P, Sepehri S, Zhou Y, et al. Development of an automated and sensitive microfluidic device for capturing and characterizing circulating tumor cells (CTCs) from clinical blood samples. PLoS One 2016;11:e0147400.

37. Hodgkinson CL, Morrow CJ, Li Y, et al. Tumorigenicity and genetic profiling of circulating tumor cells in small-cell lung cancer. Nat Med 2014;20:897-903.

38. Lim M, Cho YK. Current methods of circulating tumor cell detection. Korean J Helicobacter Up Gastrointest Res 2018;18:157-161.

39. Alix-Panabières C, Pantel K. Challenges in circulating tumour cell research. Nat Rev Cancer 2014;14:623-631.

40. Adams DL, Stefansson S, Haudenschild C, et al. Cytometric characterization of circulating tumor cells captured by microfiltration and their correlation to the CellSearch $\left({ }^{\circledR}\right)$ CTC test. Cytometry A 2015;87:137-144.

41. Gradilone A, Iacovelli R, Cortesi E, et al. Circulating tumor cells and "suspicious objects" evaluated through CellSearch ${ }^{\circledR}$ in metastatic renal cell carcinoma. Anticancer Res 2011;31:4219-4221.

42. Rosenberg R, Gertler R, Friederichs J, et al. Comparison of two density gradient centrifugation systems for the enrichment of disseminated tumor cells in blood. Cytometry 2002;49:150-158.

43. Coumans FA, van Dalum G, Beck M, Terstappen LW. Filter characteristics influencing circulating tumor cell enrichment from whole blood. PLoS One 2013;8:e61770.

44. Teo J, Mirenska A, Tan M, et al. A preliminary study for the assessment of PD-L1 and PD-L2 on circulating tumor cells by microfluidic-based chipcytometry. Future Sci OA 2017;3:FSO244.

45. Moon HS, Kwon K, Kim SI, et al. Continuous separation of breast cancer cells from blood samples using multi-orifice flow fractionation (MOFF) and dielectrophoresis (DEP). Lab Chip 2011;11:11181125.

46. Tang L, Zhao S, Liu W, et al. Diagnostic accuracy of circulating tumor cells detection in gastric cancer: systematic review and meta-analysis. BMC Cancer 2013;13:314.

47. Ghossein RA, Bhattacharya S, Rosai J. Molecular detection of micrometastases and circulating tumor cells in solid tumors. Clin Cancer Res 1999;5:1950-1960.

48. Pituch-Noworolska A, Kolodziejczyk P, Kulig J, et al. Circulating tumour cells and survival of patients with gastric cancer. Anticancer Res 2007;27:635-640.

49. Hiraiwa K, Takeuchi H, Hasegawa H, et al. Clinical significance of circulating tumor cells in blood from patients with gastrointestinal cancers. Ann Surg Oncol 2008;15:3092-3100.

50. Matsusaka S, Chin K, Ogura M, et al. Circulating tumor cells as a surrogate marker for determining response to chemotherapy in patients with advanced gastric cancer. Cancer Sci 2010;101:10671071.

51. Uenosono Y, Arigami T, Kozono T, et al. Clinical significance of circulating tumor cells in peripheral blood from patients with gastric cancer. Cancer 2013;119:3984-3991.

52. Okabe H, Tsunoda S, Hosogi H, et al. Circulating tumor cells as an independent predictor of survival in advanced gastric cancer. Ann Surg Oncol 2015;22:3954-3961.

53. Yuan D, Chen L, Li M, et al. Isolation and characterization of circulating tumor cells from human gastric cancer patients. J Cancer Res Clin Oncol 2015;141:647-660.

54. Kolostova K, Matkowski R, Gürlich R, et al. Detection and cultivation of circulating tumor cells in gastric cancer. Cytotechnology 2016;68:1095-1102.

55. Li Y, Gong J, Zhang Q, et al. Dynamic monitoring of circulating tumour cells to evaluate therapeutic efficacy in advanced gastric cancer. Br J Cancer 2016;114:138-145.

56. Kang HM, Kim GH, Jeon HK, et al. Circulating tumor cells detected by lab-on-a-disc: role in early diagnosis of gastric cancer. PLoS One 2017;12:e0180251.

57. Liu Y, Ling Y, Qi Q, et al. Prognostic value of circulating tumor cells in advanced gastric cancer patients receiving chemotherapy. Mol Clin Oncol 2017;6:235-242.

58. Pernot S, Badoual C, Terme M, et al. Dynamic evaluation of circulating tumour cells in patients with advanced gastric and oesogastric junction adenocarcinoma: prognostic value and early 
assessment of therapeutic effects. Eur J Cancer 2017;79:15-22.

59. Brungs D, Lynch D, Luk AW, et al. Cryopreservation for delayed circulating tumor cell isolation is a valid strategy for prognostic association of circulating tumor cells in gastroesophageal cancer. World J Gastroenterol 2018;24:810-818.

60. Yang C, Zhang N, Wang S, et al. Wedge-shaped microfluidic chip for circulating tumor cells isolation and its clinical significance in gastric cancer. J Transl Med 2018;16:139.

61. Jeon HK, Kim GH. Clinical significance of circulating tumor cells in gastric cancer. Korean J Helicobacter Up Gastrointest Res 2018;18:162-167.

62. Huang X, Gao P, Sun J, et al. Clinicopathological and prognostic significance of circulating tumor cells in patients with gastric cancer: a meta-analysis. Int J Cancer 2015;136:21-33.

63. Parkinson DR, Dracopoli N, Petty BG, et al. Considerations in the development of circulating tumor cell technology for clinical use. J Transl Med 2012;10:138.

64. Müller V, Stahmann N, Riethdorf S, et al. Circulating tumor cells in breast cancer: correlation to bone marrow micrometastases, heterogeneous response to systemic therapy and low proliferative activity. Clin Cancer Res 2005;11:3678-3685.

65. Pantel K, Schlimok G, Braun S, et al. Differential expression of proliferation-associated molecules in individual micrometastatic carcinoma cells. J Natl Cancer Inst 1993;85:1419-1424.

66. Ke Z, Lin M, Chen JF, et al. Programming thermoresponsiveness of NanoVelcro substrates enables effective purification of circulating tumor cells in lung cancer patients. ACS Nano 2015;9:62-70.

67. Sundaresan TK, Sequist LV, Heymach JV, et al. Detection of T790M, the acquired resistance EGFR mutation, by tumor biopsy versus noninvasive blood-based analyses. Clin Cancer Res 2016;22:1103-1110.

68. Yeo T, Tan SJ, Lim CL, et al. Microfluidic enrichment for the single cell analysis of circulating tumor cells. Sci Rep 2016;6:22076.

69. Iwatsuki M, Toyoshima K, Watanabe M, et al. Frequency of HER2 expression of circulating tumour cells in patients with metastatic or recurrent gastrointestinal cancer. Br J Cancer 2013;109:28292832. 